\title{
La radionécrose cérébrale. Limites et perspectives des modèles expérimentaux
}

\author{
J.L. LEFAIX*
}

(Manuscrit reçu le 9 octobre 1991)

RÉSUMÉ La radiothérapie, traitement efficace des tumeurs cérébrales, peut avoir plusieurs effets secondaires dont la radionécrose tardive. Les lésions de nécrose et de gliose présentent une évolution généralement irréversible dans les mois ou les années qui suivent le traitement. Plusieurs facteurs sont liés à son apparition : l'âge et l'état systémique du malade, les facteurs de risque vasculaire et la susceptibilité individuelle, mais le protocole et la dose par fraction sont essentiels, bien que des cas de radionécrose aient été décrits après radiothérapie conventionnelle. La physiopathologie de la radionécrose reste controversée malgré les théories gliales, vasculaires et autoimmunes fréquemment évoquées. Aucune théorie prise isolément ne peut expliquer la variabilité des délais d'apparition des lésions de radionécrose, la sensibilité particulière de la substance blanche, les modifications de fonction des oligodendrocytes et les anomalies vasculaires. Les lésions oligodendrogliales jouent probablement un rôle importánt au début du processus alors que l'atteinte de la barrière hémato-astrocytaire semble responsable des complications différées. Le méthodes récentes d'exploration biophysiques non invasives, comme les tomographies $\mathrm{RX}$ et par émission de positons, et l'imagerie et la spectroscopie par résonance magnétique nucléaire, ont permis l'examen anatomophysiologique du cerveau après irradiation. Les limites et perspectives de ces méthodes biophysiques sont analysées ici dans des modèles expérimentaux d'étude de la radionécrose cérébrale, développés dans des espèces variant du rongeur au primate.

ABSTRACT Cerebral radiation necrosis is the major CNS hazard of clinical treatment therapy involving delivery of high doses of radiation to the brain. It is generally irreversible and frequently leads to death from brain necrosis. Necrosis has been reported with total doses of $60 \mathrm{~Gy}$, delivered in conventional fractions. Symptoms depend upon the volume of brain irradiated and are frequently those of an intracranial mass and may be present as an area of gliosis or frank necrosis. Possible causes include some direct effect of radiation on glial cells, vascular changes and the action of an immunological mechanism. The weight of evidence suggests that demyelination is important in the early delayed reaction, and that vascular changes gradually become more important in the late delayed reactions, from several months to years after treatment. The advent of sophisticated radiographic technologies such as computed tomography, magnetic resonance imaging and spectroscopy, and positron emission tomography have facilitated serial non invasive examination of morphologic or physiologic parameters within the brain after irradiation. Limits and prospects of these technologies are reviewed in experimental animal models of late radiation injuries of the brain, which were carried out in many species ranging from mouse to monkey.

* Commissariat à l'énergie atomique, Direction des sciences du vivant, Département de pathologie et toxicologie expérimentales, Laboratoire de radiobiologie appliquée, 91191 Gifsur-Yvette Cedex. 
La radiothérapie est l'un des traitements les plus efficaces des tumeurs cérébrales, mais elle peut avoir des effets secondaires : radionécrose, démences radiques, endocrinopathies et tumeurs radioinduites. La première observation de radionécrose cérébrale retardée a été rapportée chez l'homme en 1930. Depuis, de telles lésions ont été décrites après irradiation de tumeurs intracrâniennes, épi-crâniennes, nasopharyngiennes et orbitaires.

Les lésions cérébrales apparaissent entre 1 et 3 ans. Pour un niveau de survie cellulaire critique, les plages de nécrose de coagulation et l'œdème associé se développent principalement dans la substance blanche et en un temps très court. L'ischémie due aux lésions des artérioles et du réseau capillaire joue également un rôle important dans la genèse de ces lésions. Lors d'études rétrospectives, il a été possible de distinguer les lésions précoces de nécrose de coagulation, qui apparaissent pour des doses élevées, des lésions différées d'origine vasculaire, qui apparaissent pour des doses plus faibles aussi bien dans la substance blanche que dans la substance grise. Si la dose totale dépasse $60 \mathrm{~Gy}$ administrés en 6 semaines, la probabilité de voir des complications se développer est de $5 \%$ à 5 ans pour un volume irradié supérieur à $1 / 3$ du cerveau. Cette probabilité passe à $50 \%$ à 5 ans pour l'irradiation du cerveau entier à la même dose [17]. Cependant, ces complications ne sont pas uniquement le fait d'une radiothérapie à des doses trop élevées : une chimiothérapie associée, des facteurs endocriniens et nutritifs, une pathologie vasculaire latente et l'état systémique du malade sont impliqués dans la survenue des lésions de radionécrose cérébrale retardées [32].

La physiopathologie de ces complications post-radiothérapiques reste très incertaine malgré les théories gliale, vasculaire ou immune les plus fréquemment évoquées, car celles-ci doivent expliquer la variabilité des délais d'apparition des lésions, la sensibilité particulière de la substance blanche et les anomalies vasculaires. En pratique, il est très difficile de savoir si l'aggravation de la symptomatologie neurologique témoigne d'une récidive tumorale, d'une nécrose cérébrale ou de l'association de ces deux facteurs. La tomodensitométrie $R X$, l'imagerie par résonance magnétique nucléaire (IRM) ou la tomographie par émission de positons (TEP) permettent rarement de trancher entre ces deux hypothèses $[13,15,40]$. Cependant, le pronostic d'une telle pathologie repose essentiellement sur un diagnostic rapide et sur une thérapeutique associant chirurgie et corticothérapie.

Nous rapportons ici une synthèse des travaux de recherches effectuées sur la radionécrose du système nerveux central dans différentes espèces animales, afin d'en souligner les limites et les perspectives possibles quant à une transposition méthodologique ou à une interprétation de la pathologie humaine (Tab. I). 
TABLEAU ।

Limites connues de radionécrose cérébrale dans différents modèles expérimentaux

\begin{tabular}{|c|c|c|c|c|c|c|c|c|}
\hline Espece & Age & $\begin{array}{r}\text { Energie } \\
.\end{array}$ & Vol. & $\begin{array}{c}\text { Dose } \\
\text { fractionnte } \\
\text { Gy / sernaines } \\
\end{array}$ & $\begin{array}{l}\text { Dose } \\
\text { unique } \\
\text { Gy }\end{array}$ & Méthode & Observation & Réf. \\
\hline \multirow[t]{2}{*}{ Homme } & adulte & radiotherapie & $\begin{array}{l}1 / 3 \\
2 / 3 \\
3 / 3 \\
\end{array}$ & $\begin{array}{l}d<60 / 6 \\
d<50 / 6 \\
d<45 / 6\end{array}$ & & & nécrose $5 \%$ a 5 ans & [17] \\
\hline & adulte & radiothérapie & $\begin{array}{l}1 / 3 \\
2 / 3 \\
3 / 3\end{array}$ & $\begin{array}{l}d<75 / 6 \\
d<65 / 6 \\
d<60 / 6\end{array}$ & & & nécrose $50 \%$ ans 5 ans & {$[17]$} \\
\hline Singe & $\begin{array}{l}\text { jeune } \\
\text { adulte } \\
\text { adulte }\end{array}$ & $\begin{array}{l}\text { ac. lin. } 20 \mathrm{MeV} \\
\text { ac. lin. } 20 \mathrm{MeV} \\
\text { ac. lin. } 13.2 \mathrm{MeV}\end{array}$ & $\begin{array}{l}3 / 3 \\
3 / 3 \\
3 / 3\end{array}$ & $\begin{array}{c}40 / 4<d<60 / 6 \\
d<60 / 6\end{array}$ & $10<d<15$ & $\begin{array}{l}\text { histologie } \\
\text { histologie } \\
\text { histologie }\end{array}$ & $\begin{array}{l}\text { micronécrose à } 6 \text { mois } \\
\text { micronécrose à } 6 \text { mois } \\
\text { télangiectasies à } 24 \text { mois }\end{array}$ & $\begin{array}{l}{[11]} \\
{[11]} \\
{[51]}\end{array}$ \\
\hline Chien & $\begin{array}{l}\text { jeune } \\
\text { jeune } \\
\text { adulte }\end{array}$ & $\begin{array}{l}\text { cobalt } 60 \\
\text { ac. lin. } 4 \mathrm{MeV} \\
\text { neutrons } 15 \mathrm{MeV}\end{array}$ & $\begin{array}{l}3 / 3 \\
1 / 2 \\
3 / 3\end{array}$ & $\begin{array}{l}60 / 7<d<907 \\
13 / 7<d<207\end{array}$ & $14.3<d<15$ & $\begin{array}{l}\text { histologie } \\
\text { tomographie RX } \\
\text { tomographie RX }\end{array}$ & $\begin{array}{l}\text { létal à } 6 \text { mois } \\
\text { macronécrose à } 5 \text { mois } \\
\text { létal à } 3 \text { mois }\end{array}$ & $\begin{array}{l}{[56]} \\
{[18]} \\
{[6]}\end{array}$ \\
\hline Chat & adulte & ac. lin. $6 \mathrm{MeV}$ & focal & & $d<50$ & IRM; ${ }^{31}$ P.SRM & nécrose $\mathbf{8}$ mois & [23] \\
\hline & $\begin{array}{l}\text { jeune } \\
\text { jeune } \\
\text { jeune }\end{array}$ & $\begin{array}{l}\text { betatron } 34 \mathrm{MV} \\
\mathrm{RX} 195 \mathrm{kV} \\
\text { cobalt } 60\end{array}$ & $\begin{array}{l}3 / 3 \\
3 / 3 \\
3 / 3\end{array}$ & & $\begin{array}{l}d<21 . \\
d<23 \\
d<23\end{array}$ & $\begin{array}{l}\text { histologie } \\
\text { histologie } \\
\text { histologie }\end{array}$ & $\begin{array}{l}\text { nécrose a } 9 \text { mois } \\
\text { hémorragies à } 3 \text { mois } \\
\text { nécrose à } 6 \text { mois }\end{array}$ & [24] \\
\hline Lapin & $\begin{array}{l}\text { adulte } \\
\text { jeune } \\
\text { jeune } \\
\text { jeune }\end{array}$ & $\begin{array}{l}\text { RX } 200 \mathrm{kV} \\
\text { cobalt } 60 \\
\text { hélium } 230 \mathrm{MeV} \\
\text { néon } 425 \mathrm{MeV}\end{array}$ & $\begin{array}{c}1 / 2 \\
3 / 3 \\
1 / 2 \\
\text { focal }\end{array}$ & & $\begin{array}{c}19<d<23 \\
d<16 \\
15<d<30 \\
d<20\end{array}$ & $\begin{array}{l}\text { histologie } \\
\text { histologie } \\
{ }^{18} \text { F-FDG-TEP } \\
\text { histologie }\end{array}$ & $\begin{array}{l}\text { nécrose } \\
\text { micronécrose à } 6 \text { mois } \\
\text { nécrose à } 9 \text { mois } \\
\text { micronécrose à } 6 \text { mois }\end{array}$ & $\begin{array}{l}{[45]} \\
{[52]} \\
{[34]} \\
{[53]}\end{array}$ \\
\hline Rat & $\begin{array}{l}\text { adulte } \\
\text { adulte } \\
\text { adulte } \\
\text { adulte } \\
\text { jeune }\end{array}$ & $\begin{array}{l}\text { RX } 220 \mathrm{kV} \\
\text { RX } 250 \mathrm{kV} \\
\text { RX } 300 \mathrm{kV} \\
\text { cobalt } 60 \\
\text { hélium } 230 \mathrm{MeV}\end{array}$ & $\begin{array}{l}3 / 3 \\
3 / 3 \\
3 / 3 \\
\text { tête } \\
1 / 2\end{array}$ & $d<20 / 1$ & $\begin{array}{c}10<d<20 \\
d<17.5 \\
d<11 \\
d<30\end{array}$ & $\begin{array}{l}\text { histologie } \\
\text { histologie } \\
\text { histologie } \\
\text { histologie } \\
\text { IRM: }{ }^{1} \text { H-SRM }\end{array}$ & $\begin{array}{l}\text { nécrose à } 18 \text { mois } \\
\text { lésions vasculaires a } 9 \text { mois } \\
\text { aplasie microglie à } 6 \text { mois } \\
\text { activation microglie a } 6 \text { mois } \\
\text { nécrose à } 7 \text { mois }\end{array}$ & $\begin{array}{l}{[25]} \\
{[9]} \\
{[42]} \\
{[39]} \\
{[43]}\end{array}$ \\
\hline Souris & adulte & $\mathrm{R} \times 250 \mathrm{kV}$ & $3 / 3$ & & $13<d<25$ & histologie & necrose 10 mois & [54] \\
\hline
\end{tabular}




\section{Modèles expérimentaux}

\section{Rongeurs}

L'irradiation collimatée rigoureuse du cerveau de souris est difficile techniquement en raison de sa petite taille. L'utilisation de cette espèce a donc été limitée à des études statistiques de survie après irradiation de la tête entière, avec des séries comportant plusieurs centaines d'individus $[35,55]$.

Le rat est devenu un standard de la radiobiologie du système nerveux central (SNC) depuis l'étude de Hopewell et Wright (1970) sur des cerveaux de rats normaux et hypertendus, irradiés à des doses uniques comprises entre 10 et 40 Gy (RX 220-250 kV). La période de latence étant d'autant plus courte que la dose augmente, une irradiation unique à une dose comprise entre 20 et 25 Gy semble être le seuil nécessaire pour induire des lésions conjonctivo-vasculaires tardives chez $100 \%$ des animaux $[7,8,26]$. Ces études ont été complétées, sur le même modèle expérimental, par des comparaisons de radiosensibilité de différentes souches de rats, par des résultats biochimiques [21, 22] et par des mesures de débit et de volume sanguin cérébral $[29,30]$, sur des périodes variant de 15 jours à 2 ans après irradiation. Sur le même modèle, les indices "50\% des animaux affectés" et "50\% de la période de latence", ont été utilisés pour déterminer la dose seuil de tolérance par le calcul mathématique $[9,41]$.

La radionécrosé du SNC a été largement étudiée chez le lapin depuis le travail de référence de Russel et al. en 1949 [45]. La démarche expérimentale consiste en une description macroscopique et anatomopathologique des lésions par les colorations histologiques caractéristiques du système nerveux, après observation de la période de latence clinique pour des irradiations uniques de 19 à 23 Gy ( $R X 220 \mathrm{kV})$. En raison du volume de la boîte cranienne et de son utilisation aisée en neuropathologie, le lapin restera longtemps l'animal privilégié, pour étudier les effets des variations des différents paramètres radiologiques : dose, débit de dose, énergie du rayonnement et volume de cerveau irradié $[1,5,24,52$, 53]. Aujourd'hui, cette espèce est toujours très utilisée dans des expérimentations associant imagerie et spectroscopie $R M N$, métabolisme du glucose marqué et histologie, après irradiation du cerveau [34] ou de la moelle épinière [44].

\section{Primates}

Les irradiations pratiquées sur le cerveau de primates ont débuté dans les années 1930, et ont été très développées dans les années 1950 avec la pratique d'irradiations uniques à doses souvent très élevées $[2,3,4,12,50]$. Cette espèce n'a été utilisée de manière rationnelle que depuis une quinzaine d'années, avec la pratique d'irradiations fractionnées comparables aux protocoles conventionnels de radiothérapie (accélérateurs de 10-20 MeV et doses totales de 40 Gy/4 semaines à 
60 Gy/6 semaines). II a ainsi été possible d'étudier le rôle de l'âge du sujet irradié, de l'énergie du rayonnement, de la dose et du fractionnement dans le développement des lésions cérébrales. Ces études ont été remarquablement documentées sur le plan clinique et anatomopathologique, sur le cerveau par Caveness et son groupe dans les années $1970[10-11,51]$ et sur la moelle épinière par le groupe de Schultheiss [46-47].

\section{Chien}

Le chien a été utilisé plus tardivement que le primate par Kramer et Michaelson (1972), Zook et Bradley [6,56], et le groupe de Fike dans les années $1980[18,20,48]$, aussi bien après des irradiations à doses uniques ou fractionnées qu'en curiethérapie [19, 28, 49].

\section{Limites expérimentales}

\section{Dose unique / dose fractionnée et anesthésie des animaux}

Les radionécroses cérébrales différées, décrites chez l'homme après radiothérapie en ortho ou mégavoltage ont eu pour conséquence de favoriser l'accès des irradiateurs à l'expérimentation animale. Cependant, la toxicité induite par des anesthésies répétées, indispensables pour un protocole d'irradiations fractionnées chez l'animal, a presque toujours imposé l'irradiation dose unique-cerveau entier comme protocole dans les espèces utilisées en laboratoire. Il est toutefois possible d'irradier à dose fractionnée le cerveau de rat sans anesthésie. L'étude de Mildenberger et al. (1990) en est un très bon exemple : irradiation de rats vigiles, tête entière, dose totale 20 Gy (4 Gy/5 jours) selon le protocole d'irradiation prophylactique du cerveau dans les cancers du poumon à petites cellules. L'exposition des voies digestives supérieures et des muscles masseter lors de l'irradiation de la tête entière, entraînant rapidement une dénutrition des animaux, l'étude était malheureusement arrêtée 6 mois après irradiation, alors qu'une dose unique de 20 Gy n'induit des lésions qu'au-delà du douzième mois [25].

L'utilisation des particules en radiothérapie permet l'irradiation d'un petit volume. Cependant, compte tenu de l'énergie, une irradiation à dose trop élevée peut induire rapidement des lésions de coagulation. L'étude des modifications précoces induites par l'irradiation d'un hémi-cerveau de rat à des doses uniques de 15 à $50 \mathrm{~Gy}$ (hélium, $230 \mathrm{MeV}$ ), réalisée en IRM et en spectroscopie RMN du proton [43], montre assez bien que les lésions décrites et les altérations de la barrière hémato-astrocytaire ne sont pas représentatives de la radionécrose cérébrale différée. Ces lésions correspondent à la nécrose de coagulation telle qu'elle a été décrite après $40 \mathrm{~Gy}$, dose unique, chez le rat [25]. II en est de même pour les travaux de Woodruff et al. (1988) et de Lo et al. (1991) chez le lapin. 


\section{Méthodes d'observation}

\section{Histologie}

Parmi les différentes méthodes employées pour étudier la radionécrose cérébrale, l'histologie reste la méthode de référence, car le diagnostic n'est définitivement posé qu'après observation de préparations microscopies. Cependant, les colorations histologiques spécifiques du système nerveux central, comme les hématoxylines, les imprégnations argentiques neuronales ou gliales, les colorations spécifiques des lipides, du fer, du calcium ou des différentes voies métaboliques des neuromédiateurs, n'ont apporté, à ce jour, aucun élément prédictif de la radionécrose. En revanche, parmi 11 colorations histologiques différentes, Mildenberger et al. [39] montrent l'intérêt de l'immunocytochimie et des anticorps monoclonaux anti-système majeur d'histocompatibilité, qui révèlent précocement une activation des cellules de la microglie sans aucune autre modification structurale ou métabolique.

\section{Imagerie}

La tomographie $R X$ a été la première méthode d'imagerie employée chez l'homme pour caractériser la radionécrose cérébrale $[37,38]$. Les travaux réalisés chez le chien par Fike et son groupe ont apporté une interprétation nouvelle, par une approche quantitative de l'imagerie en tomographie $R \times[18-20,48]$.

L'intérêt diagnostic du ${ }^{18} \mathrm{~F}$-fluorodéoxyglucose et de la tomographie par émission de positons ( ${ }^{18} \mathrm{~F}-\mathrm{DG}$ et TEP) a été démontré chez l'homme par Patronas et al. [40] chez 5 patients ayant subi une radiothérapie de tumeur cérébrale, dont 2 développaient une radionécrose et 3 une récurrence tumorale : le métabolisme du glucose est réduit dans le volume de radionécrose alors qu'il est augmenté dans la tumeur, par rapport au tissu sain adjacent. Le même groupe montrait, en radioautographie quantitative chez le rat, une diminution du métabolisme du ${ }^{14} \mathrm{C}-2 \mathrm{DG}$ après une irradiation à 15 Gy à dose unique [27], bien qu'à cette dose aucune lésion radioinduite ne se développe et que l'espérance de vie soit identique chez les rats témoins et irradiés. Par ailleurs, chez le lapin anesthésié, Lo et al. [34] ont montré une forte diminution du métabolisme du glucose par ${ }^{18}$ F-DG et TEP dans le volume de cerveau irradié à 30 Gy dose unique (hélium, $230 \mathrm{MeV}$ ).

L'irradiation à faible ou forte dose entraîne donc une diminution $\mathrm{du}$ métabolisme du glucose dans le SNC. Cependant, chez des sujets vigiles, la fixation du glucose marqué permet de visualiser les contributions des cellules nerveuses et gliales, alors que chez les sujets anesthésiés la visualisation des cellules gliales est fortement amplifiée du fait de l'absence de marquage des cellules nerveuses. L'anesthésie profonde imposée aux animaux pendant l'examen en TEP diminue également de façon non négligeable le flux sanguin cérébral et atténue les différences loco-régionales du métabolisme glucidique [36]. Par ailleurs, les dépôts calciques associés aux figures de gliose, caractéristiques de la nécrose 
cérébrale devraient, au contraire, augmenter la fixation du ${ }^{18} \mathrm{~F}$-DG comme cela a été montré après des lésions expérimentales dans le cerveau de rat [14].

L'utilisation de la RMN a été étendue à l'étude expérimentale de la radionécrose cérébrale depuis la première étude comparative sur 55 patients, opposant tomographie $R X$ et IRM [16]. L'intérêt de l'imagerie RMN du proton et du sodium $\left({ }^{23} \mathrm{Na}\right)$, et de la spectroscopie RMN du phosphore $\left({ }^{31} \mathrm{P}\right)$ a été étudié chez le chat après une irradiation unique de 50 Gy (accélérateur $6 \mathrm{MeV}$ ) [23]. Si la nécrose de coagulation était parfaitement révélée après injection veineuse de gadolinium (Gd-DTPA), la spectroscopie du ${ }^{31} \mathrm{P}$ et l'imagerie du ${ }^{23} \mathrm{Na}$ n'ont apporté aucune information sur le développement des lésions. En revanche, l'étude des temps de relaxation ( $T_{1}$ et $T_{2}$ ) en IRM, avec et sans injection de GdDTPA, 10 mois après l'irradiation de cerveau de lapin (hélium $230 \mathrm{MeV}$, doses uniques 15-30 Gy), fait apparaître une plus grande définition sur des images pondérées en $T_{2}$ et révèle des microlésions sur les images pondérées en $T_{1}$ [34]. De même, l'intérêt d'aborder l'étude de la dégradation des lipides complexes de la glie par la spectroscopie RMN du proton a été démontré par Richards et Budinger [43] dans I'hémi-cerveau de rat juvénile normal, après irradiation à dose unique de 10-50 Gy (hélium $230 \mathrm{MeV}$ ). Par ailleurs, ces auteurs ont observé une augmentation du signal RMN dans l'hémi-cerveau controlatéral non irradié, observation que nous avions rapportée, également en IRM, dans un modèle d'irradiation musculaire localisée chez le lapin [33].

\section{Conclusion}

La revue de ces différents modèles expérimentaux montre que les recherches expérimentales réalisées sur la radionécrose du SNC ont toujours été en aval des observations. réalisées chez l'homme, tant au niveau des descriptions cliniques, paracliniques qu'anotomopathologiques. La nécessité d'obtenir rapidement des lésions cérébrales reproductibles d'un animal à l'autre, voire d'une espèce à l'autre, impose souvent des contraintes méthodologiques multiples à l'expérimentateur : anesthésies répétées, irradiation du cerveau entier, doses uniques très au-delà des seuils connus de radiosensibilité. De ce fait, malgré des méthodes d'études très performantes, les lésions expérimentales décrites précocément après irradiation restent éloignées des lésions différées post-radiothérapiques qui se développent tardivement chez l'homme.

Aujourd'hui, la radiothérapie multi-faisceaux élève la radionécrose au rang de moyen thérapeutique lorsque I'hyperplasie d'une paroi vasculaire, induite par une irradiation unique (60 à $100 \mathrm{~Gy}$ ) et focalisée sur quelques millimètres de diamètre, est utilisée pour obtenir l'exclusion d'un angiome. Par ailleurs, l'étude physio-pathologique de la radionécrose cérébrale semble maintenant possible in vivo. Les techniques biophysiques actuelles d'exploration permettent d'aborder les réactions 
cellulaires et des dérèglements biochimiques survenant à la suite de l'irradiation : dynamiques moléculaires (IRM ${ }^{1} \mathrm{H}$ et ${ }^{23} \mathrm{Na}$ ), consommation de glucose et métabolisme énergétique ( ${ }^{18} \mathrm{~F}-\mathrm{FDG}$ et TEP), production de lactate et études de flux enzymatiques (spectroscopie RMN ${ }^{1} \mathrm{H},{ }^{31} \mathrm{P}$, ${ }^{13} \mathrm{C}$ ). Ces différentes méthodes biophysiques étant aujourd'hui appliquées à l'homme, il est urgent de réaliser sur un modèle animal unique l'étude du développement de la radionécrose cérébrale : irradiation cérébrale sur une grande espèce (primate, chien ou porc), selon un protocole conventionnel de radiothérapie, et examens fréquents des animaux, depuis l'irradiation jusqu'à l'apparition des lésions, par tomographies $R X$ et TEP, imagerie et spectroscopie RMN, complétés par une histologie moderne. Seuls des travaux de recherche sur un modèle expérimental approprié, utilisant les informations auxquelles donnent accès ces techniques d'exploration, permettront de rendre inutile la biopsie pour confirmer un diagnostic de radionécrose cérébrale différée, en même temps qu'ils apporteront des bases plus rationnelles aux traitements par la radiothérapie multi-faisceaux.

\section{Remerciements}

Nous tenons à remercier Mmes A.M. Beau et C. Jimonet pour l'aide très précieuse qu'elles nous ont apportée dans la recherche bibliographique.

\section{RÉFÉRENCES}

[1] ADOLFSON R., GOTTFRIES C.G., HASSLET O., ROOS B.E., WINBLAD B. Late effects on rabbit brain morphology and monoamine metabolites produced by ${ }^{60} \mathrm{Co}$ irradiation. Acta Radiol. Ther. Phys. Biol., 1976, 15 (5), 433-446.

[2] ARNOLD A., BAILEY P. - Alterations in the glial cells following irradiation of the brain in primates. Arch. Pathol., 1954, 57, 383.

[3] ARNOLD A., BAILEY P., HARVEY R.A. - Intolerance of the primate brainstem and hypothalamus to conventional and high energy radiations. Neurology, 1954, 4, 575.

[4] ARNOLD A., BAILEY P., LAUGHLIN J.S. - Effects of betatron radiations on the brain of primates. Neurology, 1954, 4, 165.

[5] BERG N.O., LINDGREN M. - Relation between field size and tolerance of rabbit's brain to roentgen irradiation $(200 \mathrm{kV})$ via a slit-shaped field. Acta Radiol. Ther. Phys. Biol., 1963, 1 (3), 147-168.

[6] BRADLEY E.W., DAVIS D.O., GASKILL J.W., DEYE J.A., FISHER M.P., SLOAN G.E., ROGERS C.C. - The effects of fractionated doses of fast neutrons or photons on the canine brain : evaluation by computerized tomography and evoked response recording. Int. J. Radiat. Oncol. Biol. Phys., 1980, 6, 1685-1691.

[7] CALVO W., HOPEWELL J.W., REINHOLD H.S., YEUNG T.K. - Radiation-induced damage in the choroid plexus of the rat brain : a histological evaluation. Neuropathol. Appl. Neurol., 1986, 12, 47-61. 
[8] CALVO W., HOPEWELL J.W., REINHOLD H.S., VAN DEN BERG A.P., YEUNG T.K. - Dose dependent and time dependent changes in the choroid plexus of the irradiated brain. Br. J. Radiol., 1987, 60, 1109-1117.

[9] CALVO W., HOPEWELL J.W., REINHOLD H.S., YEUNG T.K. - Time- and doserelated changes in the white matter of the rat brain after single doses of $X$ rays. Br. J. Radiol., 1988, 61, 1043-1052.

[10] CAVENESS W.F., TANAKA A., HESS K.H., KEMPER T.L., TSO M.O.M., ZIMMERMAN L.E. - Delayed brain swelling and functionnal derangement after X-irradiation of the right visual cortex in the Macaca mulatta. Radiat Res., 1974, 57, 104-120.

[11] CAVENESS W.F:, CARSTEN A.L. - Late effects of whole brain irradiation within the therapeutic range. In : "Late biological effects of ionizing radiation", Vienna, 13-17 March 1978. Vienne : AIEA, 221-239.

[12] CLEMENTE D., HOLST E.A. - Pathological changes in neurons, neuroglia, and blood-brain barrier induced by X-irradiation of heads of monkeys. A.M.A. Arch. Neurol. Psychol., 1954, 71, 66-79.

[13] CONSTINE L.S., KONSKI A., EKHOLM S., McDONALD S., RUBIN P. - Adverse effects of brain irradiation correlated with $M R$ and $C T$ imaging. Int. J. Radiat. Oncol. Biol. Phys., 1988, 15, 319-330.

[14] COOPER R.M., THURLOW G.A. - 2-Deoxyglucose uptake in the thalamus of awake rats after neocortical ablations. Exp. Neurol., 1984, 86, 261-271.

[15] CURRAN W.J., HECHT-LEVITT C., SCHUT L., ZIMMERMAN R.A., NELSON D.F. - Magnetic resonance imaging of cranial radiation lesions. Int. J. Radiat. Oncol. Biol. Phys., 1987, 13, 1093-1098.

[16] DOOMS G.C., HECHT S., BRANT-ZAWADZKI M., BERTHIAUME Y., NORMAN D., NEWTON T.H. - Brain radiation lesions : MR imaging. Radiology, 1986, 158, 149-155.

[17] EMAMI B., LYMAN J., BROWN A., COIA L., GOITEIN M., MUNZENRIDER J.E., SHANK B., SOLIN L.J., WESSON M. - Tolerance of normal tissue to therapeutic irradiation. Int. J. Radiat. Oncol. Biol. Phys., 1991, 21, 109-122.

[18] FIKE J.R., CANN C.E., DAVIS R.L., BORNICH J.K., PHILLIPS T.L., RUSSEL L.B. - Computed tomography analysis of the canine brain : effects of hemibrain $X$ irradiation. Radiat Res., 1984, 99, 294, 310.

[19] FIKE J.R., CANN C.E., PHILLIPS T.L., BERNSTEIN M., GUTIN P.H., TUROWSKI K., WEAWER K.A., HIGGINS R.J., DASILVA V. - Radiation brain damage induced by intersitial 125 , sources : a canine model evaluated by quantitative computed tomography. Neurosurgery, 1985, 16 (4) 530-537.

[20] FIKE J.R., CANN C.E., TUROWSKI K., HIGGINS R.J., CHAN A.S.L., PHILLIPS T.L., DAVIS R.L. - Radiation dose response of normal brain. Int. J. Radiat. Oncol. Biol. Phys., 1988, 14, 63-70.

[21] GERBER B., DEROO J., BESSEMANS B., REINHOLD H.S., VERWEY-VERSTEEG A.A.C. - Late effects in the central nervous system. A study of biochemical alterations after local exposure of the rat brain to $2 \mathrm{krd}$. Strahlentherapie, 1976, 6 , 530-540.

[22] GERBER B., DEROO J., MAES J., CASALE J. - Late changes in biochemistry and blood flow in rat brain locally exposed to 30,40 or 60 Gy. Acta Radiol. Oncol., 1979, 18 (4), 376-384.

[23] GROSSMAN R.I., HECHT-LEAVITT C.M., EVANS S.E., LENKINSKI R.E., HOLLAND G.A., VAN WINKLE T.J., MCGRATH J.T., CURRAN W.J., SHETTY A. JOSEPH P.M. - Experimental radiation injury : combined MR imaging and spectroscopy. Radiology, 1988, 169, 305-309. 
[24] HASSLER O., MOVIN A. - Microangiographic studies on changes in the cerebral vessels after irradiation. I. Lesions in the rabbit produced by ${ }^{60} \mathrm{Co} \gamma$-rays, $195 \mathrm{kV}$ and 34 MV roentgen rays. Acta Radiol. Ther. Phys. Biol., 1966, 4, 279-288.

[25] HOPEWELL J.W., WRIGHT E.A. - The nature of latent cerebral irradiation damage and its modification by hypertension. Br. J. Radiol., 1970, 43, 161-167.

[26] HOPEWELL J.W. - The late vascular effets of radiation. Br. J. Radiol., 1974, 47, 158-161.

[27] ITO M., PATRONAS N.J., DI CHIRO G., MANSI L., KENNEDY C. - Effect of moderate level $X$-radiation to brain on cerebral glucose utilization. J. Comput. Assist. Tomogr., 1986, 10 (4), 584-588.

[28] JANZER R.C., KLEIHUES P., OSTERTAG C.B. - Early and late effects on the normal dog brain of permanent intersitial iridium 192 irradiation. Acta Neuropathol., 1986, $70(2), 91-102$.

[29] KEYEUX A., DUNJIC A., ROYER E., JOVANOVIC D., VAN DE MERCKT J. Late functional and circulatory changes in rats after local irradiation. Int. J. Radiat. Biol., 1971, 20 (1), 7-25.

[30] KEYEUX A. - Late modifications of cephalic circulation in head $X$-irradiated rats. Radiat. Environ. Biophys., 1976, 13, 125-135.

[31] KRAMER M.W., MICHAELSON S.M. - Late pathophysiologic changes in head Xirradiated dogs ; review and clinical correlations. Radiat. Res., 1972, 49, 563-588.

[32] LEIBEL A., SHELINE G.E. - Tolerance of the central and peripheral nervous system to therapeutic irradiation. Adv. Radiat. Biol., 1987, 12, 257-288.

[33] LEFAIX J.L., DABURON F., MARTIN J.L., JEANDEY C. - Intérêt de l'analyse en composantes principales appliquée à l'imagerie RMN pour le diagnostic précoce de l'irradiation aiguë localisée. Etude expérimentale chez le lapin. Innov. Technol. Biol. Méd., 1988, 9 (4), 429-445.

[34] LO E.H., DELAPAZ R.L., FRENKEL K.A., POLJAK A., PHILLIPS M.H., BRENNAN K.M., WOODRUFF K.H., VALK P.E., STEINBERG G.K., FABRIKANT J.I. - MRI and PET of delayed heavy-ions radiation injury in the rabbit brain. Int. J. Radiat. Oncol. Biol. Phys., 1991, 20, 689-696.

[35] MASON H.C., MASON B.T., MOOS W.S. - Total-head (brain) $X$ irradiation of mice and primary factors involved. Brit. J. Radiol., 1955, 28 (333) 495-507.

[36] MIES G., NIEBUHR I., HOSSMANN K.A. - Simultaneous measurement of blood flow and glucose metabolism by autoradiographic techniques. Stroke, 1981, 12 (5), 581-588.

[37] MIKHAEL M.A. - Radiation necrosis of the brain : correlation between computed tomography, pathology, and dose distribution. J. Comput. Assist. Tomogr., 1978, 2, 71-80.

[38] MIKHAEL M.A. - Radiation necrosis of the brain : correlation between patterns in computed tomography and dose distribution. J. Comput. Assist. Tommogr., 1978, 2, 241-249.

[39] MILDENBERGER M., BEACH T.G., MCGEER E.C., LUDGATE C.M. - An animal model of prophylactic cranial irradiation : histologic effets at acute, early and delayed stages. Int. J. Radiat. Oncol. Biol. Phys., 1990, 18, 1051-1060.

[40] PATRONAS N.J., DI CHIRO G., BROOKS R.A., DE LA PAZ R.L., KORNBLITH P.L., SMITH B.H., RIZZOLI H.V., KESSLER R.M., MANNING R.G., CHANNING M., WOLF A.P., O'CONNOR C.M. - Work in progress: [18F] fluorodeoxyglucose and positron emission tomography in the evaluation of radiation necrosis of the brain. Radiology, 1982, 144, 885-889.

[41] REINHOLD H.S., CALVO W., HOPEWELL J.W., VAN DEN BERG A.P. Development of blood vessel-related radiation damage in the fimbria of the central nervous system. Int. J. Radiat. Oncol. Biol. Phys., 1990, 18, 37-42. 
[42] REYNERS H., GIANFELICI DE REYNERS E., MAISIN J.R. - Effets tardifs de l'irradiation sur l'ultrastructure du cortex cérébral chez le rat. C.R. Soc. Biol., 1979, 173, 669-676.

[43] RICHARDS T., BUDINGER T.F. - NMR Imaging and spectroscopy of the mammalian central nervous system after heavy ion radiation. Radiat. Res., 1988, 113, 79-101.

[44] RUBIN P., WHITAKER J.N., CECKLER T.L., NELSON D., GREGORY P.K., BAGGS R.B., CONSTINE L.S., HERMAN P.K. - Myelin basic protein and magnetic resonance imaging for diagnosing radiation myelopathy. Int. J. Radiat. Oncol. Biol. Phys., 1988, 15, 1371-1381.

[45] RUSSEL D.S., WILSON C.M., TANSLEY K. - Experimental radionecrosis of the brain in rabbits. J. Neurosurg. Psychol., 1949, 12, 187-195.

[46] SCHULTHEISS T.E., STEPHENS L.C., JIANG G.L., ANG K.K., PETERS L.J. Radiation myelopathy in primates treated with conventional fractionation. Int. J. Radiat. Oncol. Biol. Phys., 1990, 19, 935-940.

[47] STEPHENS L.C., HUSSEY D.H., RAULSTON G.L., JARDINE J.H., GRAY K.N., ALMOND P.R. - Late effects of $50 \mathrm{MeV}$ neutron and colbalt-60 irradiation of rhesus monkey cervical spinal cord. Int. J. Radiat. Oncol. Biol. Phys., 1983, 9, 859-864

[48] TILLER-BORCICH K., FIKE J.R., PHILLIPS T.L., DAVIS R.L. - Pathology of delayed radiation brain damage : an experimental canine model. Radiat. Res., $1987,110,161-172$.

[49] TUROWSKI K., KIKE J.R., CANN C.E., HIGGINS R.J., DAVIS R.L. GUTIN P.H., PHILLIPS T.L., WEAVER K.A.. - Normal brain iodine-125 radiation damage : effect of dose and irradiated volume in a canine model. Radiology, 1986, 833-838.

[50] VOGEL F.S., PICKERING J.E. - Demyelinization induced in the brains of monkeys by means of fast neutrons. Pathogenesis of the lesion and comparison with the lesions of multiple sclerosis and Schilder's disease. J. Exp. Med., 1956, 104 (3), 435-449.

[51] WAKISAKA S., O'NEILL R.R., KEMPER T.L., VERRELLI D.M., CAVENESS W.F. - Delayed brain damage in adult monkeys from radiation in the therapeutic range. Radiat. Res., 1979, 80, 277-291.

[52] WIBERG A., ADOLFSON R., ECKERT B., WINBLAD B. - The activity of monoamine oxidases $A$ and $B$ in gamma-irradiated rabbit brains. Experientia, 1982, 38, 71-73.

[53] WOODRUFF K.H., LYMAN J.T., FABRIKANT J.L. - Heavy charged-particle induced lesions in rabbit cerebral cortex. Int. J. Radiat. Oncol. Biol. Phys., 1988, 14, 301-307.

[54] YOSHII Y., MAKI Y.. - Late radiation effects in the mouse brain, a quantitative study on PAS positive deposits. Acta Oncol., 1987, 26 (5), 373-376.

[55] YOSHII Y., MAKI Y., TSUNEMOTO H., KOIKE S., KASUGA T. - The effect of acute total-head $X$ irradiation on $\mathrm{C} 3 \mathrm{H} / \mathrm{He}$ mice. Radiat. Res., 1981, 86, 152-170.

[56] ZOOK C., BRADLEY E.W., CASARETT G.W. ROGERS C.C. - Pathologic findings in canine brain irradiated with fractionated fast neutrons or photons. Radiat. Res., 1980, 84, 562-578. 